REFERENCE

1. Miles J, Ballance K. Omalizumab: the patient experience. Am J Respir Crit Care Med 2010;181:A1339.

\section{P109 INTRAVENOUS AMINOPHYLLINE IN THE TREATMENT OF SEVERE ASTHMA: DO WE NEED ECG MONITORING?}

doi:10.1136/thoraxjnl-2011-201054c.109

J G G B Penge, J Hull, K Guha, A Menzies-Gow. Royal Brompton and Harefield NHS Foundation Trust, London, UK

Introduction Intravenous (IV) aminophylline has an established role in the management of acute severe asthma, with beneficial bronchodilatory, anti-inflammatory and immunomodulatory effects. However, concern is often raised regarding its potential to prolong the OT interval and precipitate cardiac arrhythmias, given its catecholamine enhancing effect. ${ }^{1}$ Despite this, the influence of IV aminophylline on electrocardiographic (ECG) parameters in patients with acute severe asthma is not fully known. We routinely perform 12-lead electrocardiography on all patients before and $24 \mathrm{~h}$ after commencing IV aminophylline.

Objective To evaluate the effect of intravenous aminophylline on ECG parameters in patients with acute severe asthma.

Method We assessed electrocardiographic parameters in 26 (21 female) consecutive patients, with a mean (SD) age of 48 (11) years, admitted with severe asthma. We measured serum electrolytes, aminophylline levels and ECG parameters at baseline and within $48 \mathrm{~h}$ of commencing IV aminophylline. ECGs were independently assessed by a cardiologist blinded to patient identity and ECG timings.

Results No significant difference in mean QTc, PR or ORS interval was found following treatment (Abstract P109 table 1). Furthermore, there was no difference in number of atrial or ventricular ectopics, T-wave amplitude or frequency of arrhythmias. However, in four patients the QTc was prolonged above the upper limit of normal ( $447 \mathrm{~ms}$ to $519 \mathrm{~ms}, 450 \mathrm{~ms}$ to $516 \mathrm{~ms}, 516 \mathrm{~ms}$ to $568 \mathrm{~ms}$ and $471 \mathrm{~ms}$ to $548 \mathrm{~ms})$.

\section{Abstract P109 Table 1}

\begin{tabular}{lccc}
\hline & Baseline & IV Aminophylline & p Value \\
\hline OTc (ms) & $460(36.2)$ & $466(46.7)$ & 0.49 \\
QRS duration (ms) & $84(7.0)$ & $84(9.1)$ & 1.00 \\
PR (ms) & $145(20.6)$ & $142(16.3)$ & 0.13 \\
Aminophylline level (mg/l) & $4.6(4.4)$ & $10.6(2.2)$ & $<0.001$ \\
Serum K+ (mmol/l) & $4.1(0.4)$ & $4.0(0.3)$ & 0.19 \\
\hline
\end{tabular}

Data as mean (SD).

Conclusions These findings are largely reassuring with regard to the safety of IV aminophylline in severe asthma, in terms of its effects on standard electrocardiographic variables and the incidence of arrhythmias. There was, however, evidence of OTc prolongation in four subjects indicating a need for caution and ECG checks, especially with concomitant use of QT prolonging drugs.

\section{REFERENCE}

1. RuDusky BM. Aminophylline: exploring cardiovascular benefits vs medical malcontent. Angiology 2005;56:295-304.

\section{P110 FLUTICASONE PROPIONATE/FORMOTEROL FUMARATE COMBINATION THERAPY IS MORE EFFICACIOUS IN IMPROVING LUNG FUNCTION THAN ITS INDIVIDUAL COMPONENTS IN PATIENTS WITH ASTHMA}

doi:10.1136/thoraxjnl-2011-201054c.110

${ }^{1} \mathrm{D}$ Price, ${ }^{2} \mathrm{~A}$ Papi, ${ }^{3} \mathrm{~K}$ Kaiser, ${ }^{4} \mathrm{~B}$ Grothe, ${ }^{4} \mathrm{M}$ Lomax, ${ }^{4} \mathrm{~T}$ Mclver. ${ }^{1}$ Centre of Academic Primary Care, University of Aberdeen, Aberdeen, UK; ${ }^{2}$ University of Ferrara, Ferrara,
Italy; ${ }^{3}$ SkyePharma, Muttenz, Switzerland; ${ }^{4}$ Mundipharma Research Limited, Cambridge, UK

Introduction and Objectives Combination therapy with an inhaled corticosteroid (ICS) and a long-acting $\aleph_{2}$-agonist (LABA) is the most effective treatment option for patients with asthma uncontrolled with ICS monotherapy. ${ }^{1}$ In practice, asthma symptoms remain uncontrolled or only partly controlled in many patients. A new therapy option for asthma combining the ICS fluticasone propionate (FLUT) and the LABA formoterol fumarate (FORM) in a single aerosol inhaler (FLUT/FORM; flutiform $^{\circledR}$ ), has been developed at doses of 100/10, 250/10 and 500/20 $\mu \mathrm{g}$ for twice-daily administration. In this analysis, data were pooled from up to five randomised, double-blind, parallel-group studies in order to assess the efficacy of FLUT/FORM in terms of improvement in lung function (as indicated by change in $\mathrm{FEV}_{1}$ ).

Methods The analysis included adults and adolescents with a range of asthma severities who were treated for 8 or 12 weeks with FLUT/ FORM $(100 / 10,250 / 10$ or $500 / 20 \mu \mathrm{g}$ twice daily) or the equivalent nominal dose of FLUT monotherapy $(100,250$ or $500 \mu \mathrm{g}$ twice daily; five studies) or FORM monotherapy $(10 \mu \mathrm{g}$ twice daily; three studies).

Results FLUT/FORM was associated with a significantly greater improvement in lung function, as indicated by change in $\mathrm{FEV}_{1}$, compared with its individual components (Abstract P110 table 1). FLUT/FORM was superior to FORM with regard to change in predose $\mathrm{FEV}_{1}$ from baseline to study end; the least-squares (LS) mean difference for FLUT/FORM vs FORM was 0.131 (95\% CI 0.07 to $0.19 ; \mathrm{p}<0.001)$. Similarly, FLUT/FORM was superior to FLUT with regard to change in $\mathrm{FEV}_{1}$ from pre-dose at baseline to $2 \mathrm{~h}$ post-dose at study end (LS mean difference FLUT/FORM vs FLUT 0.15 L [95\% CI 0.10 to $0.19 ; \mathrm{p}<0.001]$ )

Conclusions The combination of fluticasone/formoterol in a single aerosol inhaler is more effective than fluticasone or formoterol given alone in improving lung function for patients with a range of asthma severities.

Abstract P110 Table 1

FLUT/FORM vs FORM*

\begin{tabular}{lll}
\hline & FLUT/FORM (N=341) & FORM (N=345) \\
\hline $\begin{array}{l}\text { Mean FEV }{ }_{1} \text { at baseline, L (SD) } \\
\text { Pre-dose at study end }\end{array}$ & $2.33(0.60)$ & $2.34(0.62)$ \\
$\mathrm{N}$ & 341 & 345 \\
$\quad$ Mean FEV $1, \mathrm{~L}(\mathrm{SD})$ & $2.53(0.71)$ & $2.4(0.76)$ \\
$\mathrm{LS}$ mean change, L (95\% Cl)† & $0.19(0.15$ to 0.24$)$ & $0.06(0.02$ to 0.10$)$
\end{tabular}

\section{FLUT/FORM vs FLUT $\ddagger$}

\begin{tabular}{lll}
\hline & FLUT/FORM (N=641) & FLUT (N=643) \\
\hline $\begin{array}{l}\text { Mean FEV } 1 \text { at baseline, L (SD) } \\
2 \text { h post-dose at study end }\end{array}$ & $2.11(0.62)$ & $2.11(0.65)$ \\
$\mathrm{N}$ & 629 & 613 \\
$\quad$ Mean FEV $1, \mathrm{~L}(\mathrm{SD})$ & $2.52(0.78)$ & $2.38(0.75)$ \\
$\mathrm{LS}$ mean change, L $(95 \% \mathrm{Cl}) \dagger$ & $0.41(0.38$ to 0.44$)$ & $0.27(0.23$ to 0.30$)$
\end{tabular}

Study end was at week 8 or 12

Analysis of covariance was calculated with treatment and baseline pre-dose forced expiratory volume at $1 \mathrm{~s}$ as covariates, and centre as a random effect.

* Pooled analysis from three studies to include only studies with treatment arms of equivalent nominal doses to FLUT/FORM

†Least-squares mean change from baseline.

$\ddagger$ Pooled analysis from five studies to include only studies with treatment arms of equivalent nominal doses to FLUT/FORM.

FLUT, fluticasone propionate; FORM, formoterol fumarate.

\section{REFERENCE}

1. GINA. GINA report Global strategy for asthma management and prevention. 2009. http://www.ginasthma.org. 